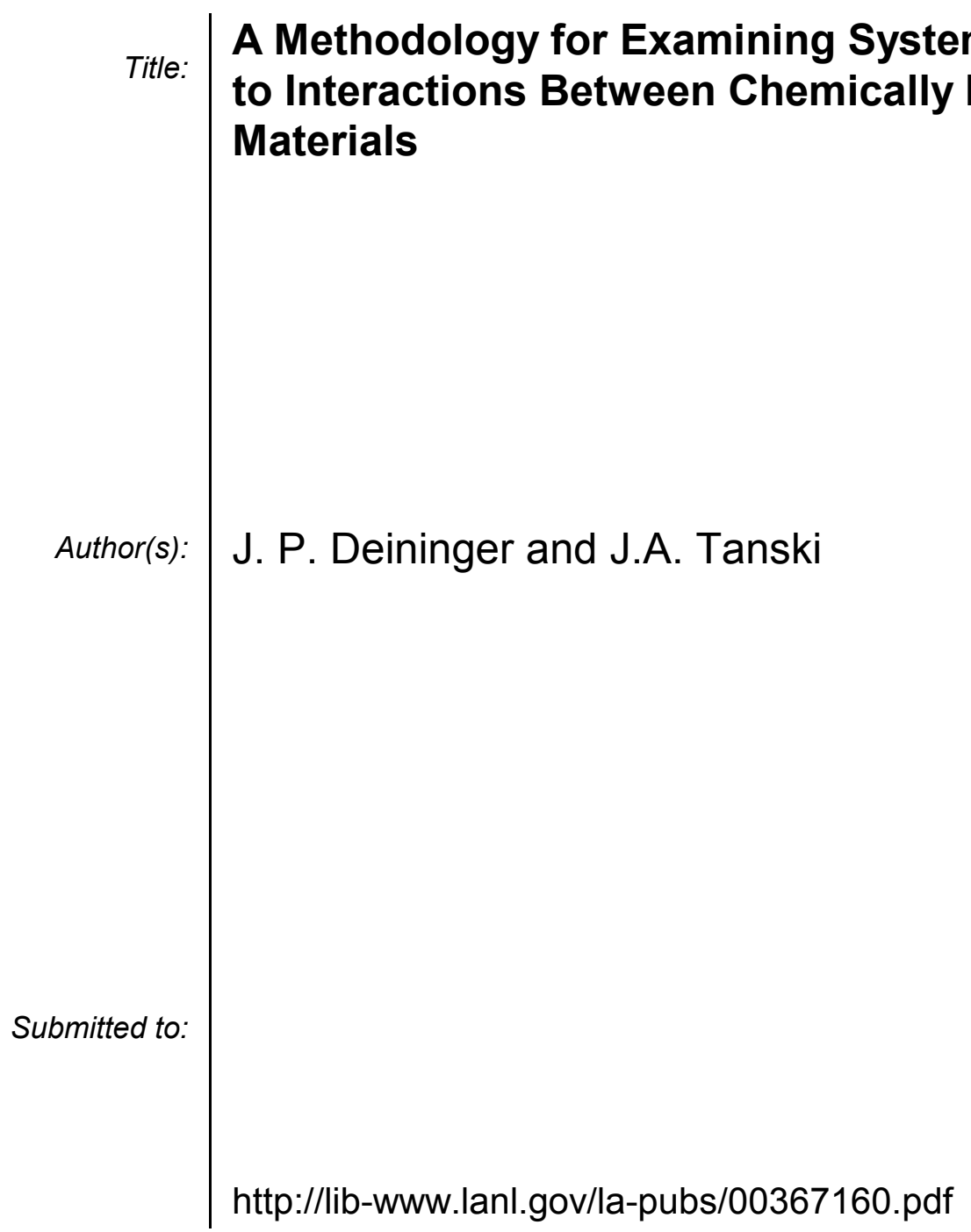

Los Alamos National Laboratory, an affirmative action/equal opportunity employer, is operated by the University of California for the U.S. Department of Energy under contract W-7405-ENG-36. By acceptance of this article, the publisher recognizes that the U.S. Government retains a nonexclusive, royaltyfree license to publish or reproduce the published form of this contribution, or to allow others to do so, for U.S. Government purposes. Los Alamos National Laboratory requests that the publisher identify this article as work performed under the auspices of the U.S. Department of Energy. Los Alamos National Laboratory strongly supports academic freedom and a researcher's right to publish; as an institution, however, the Laboratory does not endorse the viewpoint of a publication or guarantee its technical correctness. 


\title{
A Methodology for Examining System Aging Due to Interactions Between Chemically Incompatible Materials
}

\author{
J. P. Deininger and J.A. Tanski \\ Los Alamos National Laboratory, Engineering Sciences and Applications Division, Los Alamos, NM 87545
}

\begin{abstract}
We start with a stored and unused population of fielded engineered units that are composed of chemically incompatible materials. The units age primarily through heterogeneous chemical reactions between the materials resulting in possible degradation in performance. The engineered units are unused in storage, but may be called into actual service at any time. We sample several units from the population per year and perform a number of non-destructive evaluation (NDE) techniques, such as radiography, low-frequency vibration analysis, and ultrasonic imaging on the selected units. From those units, some are selected for destructive testing (D-test) involving disassembly and testing of internal parts and components. Chemical analyses, mechanical properties measurements and other tests are performed. All of the above steps provide information that is used in the system simulation mathematical model. The system simulation model incorporates chemical reactions and gassolid transport processes, along with changes in both the surface and bulk properties of the solids. Model results are used to suggest improvements in NDE analyses of the units and improvements in component and material analyses. Model results give trending indications of individual component and overall system changes over time, plus some understanding of the mechanisms involved which allow science-based predictions of the aged state of the units in future times. The NDE, D-test, and model results can also be used to assess statistically the reliability and performance of the overall aging population of units.
\end{abstract}

\section{INTRODUCTION}

There are thousands of devices and products made and distributed around the world by industrial society. It is an empirical observation that the vast majority of these products begin to change from the day that they are released from manufacture, that many retain their design functionality for a very long time measured in years or centuries, that many lose their design functionality in a very short time measured in a few hours or days, and that many simply degrade in functionality at rates anywhere between these extremes. There are many mechanisms by which these degradations occur. A particular subset exists, however, in which changes in the devices and products occur primarily due to chemical reactions which take place between the various "chemically incompatible" materials which are in close proximity to each other within the device or system. Other effects such as mechanical damage due to stress or internal material changes in phase or structure will occur, of course, but in these 
systems the fastest rate of change is due to chemical interaction between the different materials constituting the various parts and subassemblies.

Many examples of these kinds of products, devices or systems can be stated. Pyrotechnic devices, for example, consist of strong oxidizers and strong reducing agents in close proximity to each other, even sometimes within the same molecule. Over time, these materials react slowly with each other to various extents, thus leading to degradation in performance when the fireworks are displayed on July 4 after storage in a warehouse for several years since being made. Other examples include pharmaceutical, nutritional, agricultural or food products in which chemical reactions between primary chemicals and their binders or bulk carrier materials can occur over time, or published materials in which the various chemicals which make up the papers, adhesives, inks, or packaging materials can react with each other over time. Electronic products, which depend for function on electron movement properties of the various parts and contiguous materials, will lose function in storage due to slow chemical reactions between metal, plastic, and ceramic materials. Mechanical devices which in normal use would lose their functionality due to mechanical stress, frictional stress, or thermal degradation will nonetheless lose functionality in storage due to chemical reactions between lubricants and adhesives and metal or plastic components, or oxidative corrosion of the metal parts which increase the rates of these stated reactions. Automobile clutch assemblies, for instance, are stored in inventory as separate unassembled parts in closed packages, primarily because if the clutch was stored as an assembled unit, its functionality would decrease much more rapidly due to corrosion of metal parts in the presence of organic fluids and air.

Chemically incompatible materials use in products and devices, while necessary for function, is minimized in design in order to increase both the shelf life and the operating life of the products. Their use is unavoidable, however, and thus some of the primary challenges associated with these products are to understand the mechanisms which control chemical reaction changes in the products, to understand the rates at which they occur, and to understand their impact on the performance reliability of the devices over periods of time. The methodology described here has been developed to help address these challenges.

\section{METHODOLOGY}

An illustration of some of the system aging events which may occur in these types of products, devices or systems is given in Figure 1. At the time of manufacture, shown as (a), a device or product may contain parts of say, Six (6) different kinds of materials which have varying chemical reactivities with respect to each other. The parts may have static structural functions, or dynamic mechanical functions, or functions which depend upon other properties of the material from which they are made. If the parts are solid phase, they are spatially near or contiguous to each other, thereby offering the opportunity for solid-to-solid chemical reactions, or gas-to-solid chemical reactions to occur. If liquid materials are present, the higher reaction rates due to liquid phase mobility compared to solid phase might be expected. The units are stored in inventory for a long period of time under storage 
conditions which are characterized by varying environmental conditions. Examples of three different kinds of changes due to chemical behavior in the system are illustrated in (b). Chemical interaction between materials 1,2 and 4 with material 3 may cause the part made of material 3 to significantly shrink with time. This leaves gaps or separations between the four parts, allowing gas phase species to accumulate in these areas, and may reduce system functionality significantly. Oxidation-reduction reactions or electrochemical corrosion reactions between material 6 and the container material itself may lead to material removal, movement, leakage, and may reduce system functionality significantly. Chemical interaction between the parts of materials 5 and 6 , which normally were contiguous to each other but not attached, can lead to either physical or chemical binding of the two to each other, which may reduce system functionality significantly. The combined effect of these chemical behaviors, while individual effects of each may be small, is to move the actual performance of the system, device or product away from its design intent. The actual effect may be anywhere from slight performance degradation to complete performance failure.

\section{Chemical degradation of Material 3 by species from Materials 1,2 and 4 resulting in Material 3 shrinkage, new gas phase species in gaps and system functionality reduction}


$\checkmark \quad$ Static or mechanical parts,

Gas, liquid or solid phase materials

Chemical interactions of Materials 5 and 6 results in binding up of mechanical parts, reducing mechanical functionality.

Structural, mechanical, electronic, optical or chemical function
Redox chemical corrosion of Material 6 with container wall, resulting in leak and material movement, reducing functionality

Figure 1. Illustration of System Aging Events That May Lead to Reduced System Function

A new methodology for assessing the reliability and performance impacts for this class of devices is illustrated in Figure 2. A large population of devices is stored in inventory until needed under environmental conditions which are approximately known. Several of the units are randomly chosen for examination. All of these units undergo a battery of non- 
destructive tests, including internal imaging such as x-ray radiography or ultrasonic imaging, low-frequency vibration testing to get a signature of whether or not the parts are in their properly installed places and functions, and other tests. The intent here is to get as much information about the sampled units as possible without damage to any of the units. Analysis of the data is used to select one or more units for destructive testing. These chosen units are disassembled carefully, parts examined, and detailed chemical and mechanical measurements and tests are performed on the various parts. The resulting data from this work plus the non-destructive data are compared to baseline data from new units off the manufacturing line to look for changes which may occur during inventory storage [Deininger and Reader 1987]. All of these data are used as inputs into a system simulation mathematical model.

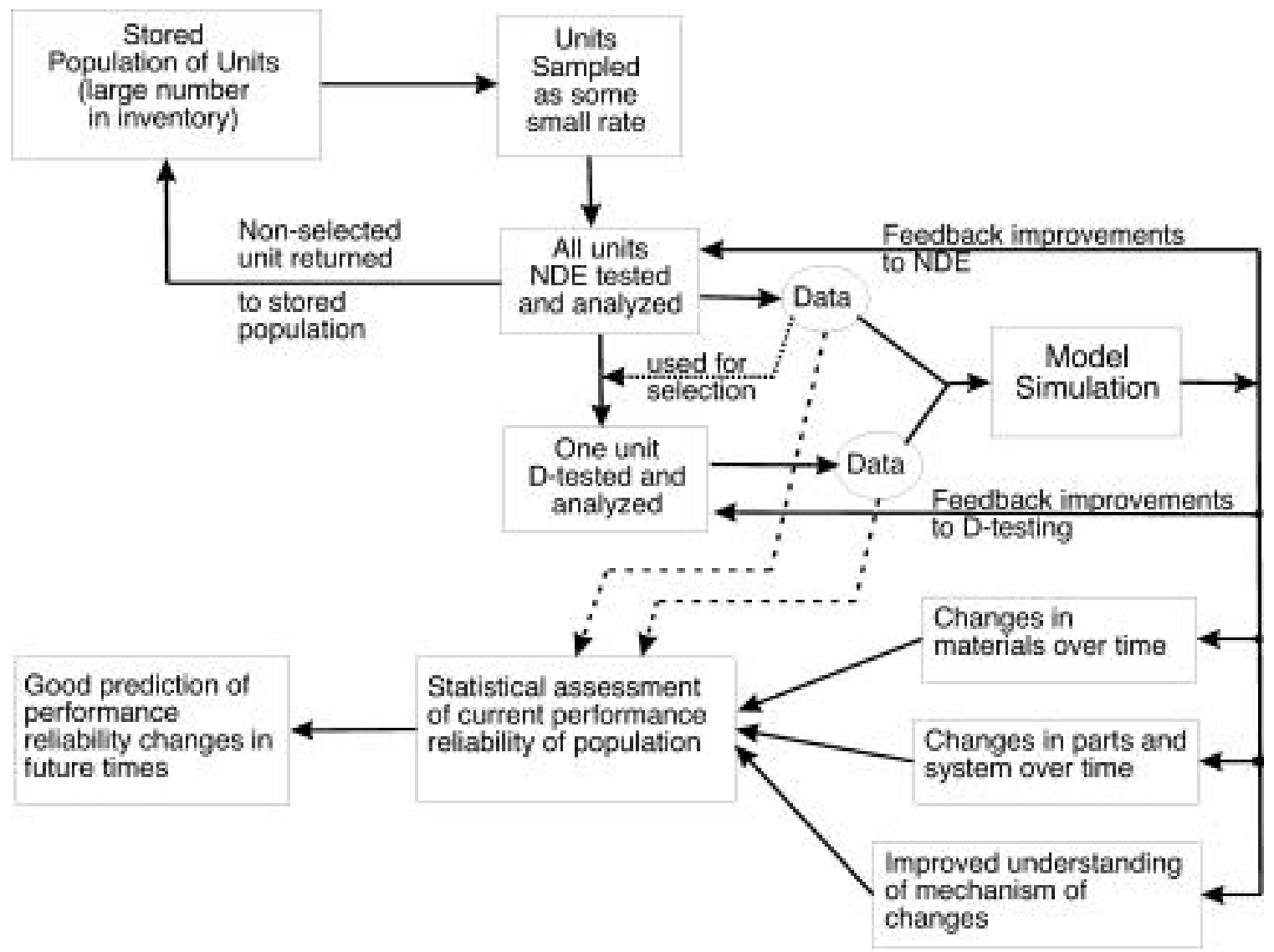

Figure 2. Illustration of Methodology 


\section{SYSTEM SIMULATION}

The system simulation model incorporates features such as known gas-solid, gas-phase and solid-solid chemical reactions, hydrodynamic gas transport through gaps and separations between parts, intrapart material species diffusion, and known phenomena such as reactive surface coatings and the like.

The fidelity of the simulation is very dependent on the degree of completeness of the numerical solution, especially in converting the real problem to its numerical equivalent. In addition, the cost (money and time) of obtaining and using a high-fidelity representation must be considered. A simulation which accurately contains all the minute features of the system but requires weeks of computational time on a supercomputer is not a desirable situation, nor is a simulation which can be computed in a matter of minutes but has such a coarse numerical geometry that it cannot accurately represent the system.

Our current approach is to use commercial software that meets the requirements of generating high-fidelity computational meshes, contains algorithms for the current physics and chemical kinetics, and provides flexibility in tailoring the software to include the unique physics in our systems. These commercial programs include FLUENT, FIDAP, and CFX2000. Each program has strengths based on their implementation of approximations of the physics, such as anisotropic k- $\varepsilon$ turbulence model [FIDAP 1999] versus algebraic Reynold stress model [FLUENT 1999] for simulating flow with high curvature geometries, or unstructured [GAMBIT 1999] versus multiblock grids for the geometric description of complex assemblages.

The numerical results obtained from the simulations are used to help corroborate the NDE and D-test results, to help understand the origins, mechanisms and causes of the changes in parts, materials and system in better detail, and over time. These results also help via feedback to suggest improvements to the NDE and D-testing regimes used, to improve the usefulness of the data which emerges, both for the simulation and for assessment of system changes. Finally, the NDE and D-testing data and the simulation results from a series of units are used to perform statistical assessments of the effects of these changes on current performance reliability of the unit population. They are also used to help predict the trends in the rate of these changes, such that accurate prediction of performance reliabilities of the unit population in future times can be completed with high confidence.

\section{CONCLUSION}


The new methodology described herein has shown itself to be an excellent new tool for assessment of the effects of long-term storage on the design performance of devices which contain parts made of inherently chemically incompatible materials. The process is undergoing continuous improvement as the feedback loops shown in Figure 2 are utilized to improve the types and details of NDE and D-test measurements and tests which are performed on selected stored units. The method has promise not only of increasing understanding of the mechanisms involved in the changes, but also of becoming a good predictive tool for trending of changes in the future and their effect on design performance of the stored devices.

\section{ACKNOWLEDGEMENTS}

This paper is based on work LA-UR-99-3453 performed at the Los Alamos National Laboratory for the U.S. Department of Energy under contract DE-AC04-94AL85000.

\section{REFERENCES}

Deininger, J. Paul and Reader, Garth, "Improved Component Selection Rationale for Weapon Surveillance" (U), Proceedings of the $21^{\text {st }}$ Aging, Compatibility and Stockpile Stewardship Conference, September 30-October 2, 1997, Albuquerque, NM, published by Sandia National Laboratory, Albuquerque, NM 87185 under Department of Energy Contract DE-AC04-94AL85000.

FIDAP Users Manual, Version 8.0, FLUENT Inc., Lebanon NH, 1999.

FLUENT5 Users Manual, Version 5.1, FLUENT Inc., Lebanon, NH, 1999.

GAMBIT Users Manual, Version 1.4, FLUENT Inc, Lebanon, NH, 1999. 